TRANSACTIONS OF THE

AMERICAN MATHEMATICAL SOCIETY

Volume 358, Number 6, Pages 2399-2413

S 0002-9947(05)03970-X

Article electronically published on December 20, 2005

\title{
ON THE CONTACT GEOMETRY OF NODAL SETS
}

\author{
R. KOMENDARCZYK
}

\begin{abstract}
In the 3-dimensional Riemannian geometry, contact structures equipped with an adapted Riemannian metric are divergence-free, nondegenerate eigenforms of the Laplace-Beltrami operator. We trace out a twodimensional consequence of this fact: there is a close relationship between the topology of the contact structure on a convex surface in the 3-manifold (the dividing curves) and the nodal curves of Laplacian eigenfunctions on that surface. Motivated by this relationship, we consider a topological version of Payne's conjecture for the free membrane problem. We construct counterexamples to Payne's conjecture for closed Riemannian surfaces. In light of the correspondence between the nodal lines and dividing curves, we interpret the conjecture in terms of the tight versus overtwisted dichotomy for contact structures.
\end{abstract}

\section{INTRODUCTION}

1.1. Payne's conjecture. If we think of a given Riemannian surface $\left(\Sigma, g_{\Sigma}\right)$ as a vibrating membrane with $u(\mathbf{x}, t), \mathbf{x} \in \Sigma$, the displacement of the membrane from the original position at time $t, u$ is a solution to the wave equation

$$
\partial_{t t} u=\Delta_{\Sigma} u \text {. }
$$

Since the solution is separable, i.e. $u(t, \mathbf{x})=v(t) w(\mathbf{x})$, we obtain an equivalent system of equations $\partial_{t t} v=\lambda v$ and $\Delta_{\Sigma} w=\lambda w(\lambda \in \mathbb{R})$. Therefore, the "stagnation points" on the membrane are exactly zeros of the eigenfunction $w$. This zero set, $\Xi(w):=\{\mathbf{x} \in \Sigma: w(\mathbf{x})=0\}$, is called a nodal set and forms interesting patterns, as originally studied by E. Chladni in the 18 th century. In the case when the membrane is a closed surface we refer to the problem (1) as the free membrane problem, and for surfaces with boundary and Dirichlet boundary conditions we refer to the problem as the fixed membrane problem (see [6]). For an arbitrary smooth Riemannian surface $\left(\Sigma, g_{\Sigma}\right)$ nodal sets have been characterized by S. Cheng in [6], where it is proved that the nodal set is a collection of $C^{2}$-immersed closed curves in $\Sigma$. For a generic metric, K. Uhlenbeck [28] showed that these curves are embedded circles with no critical points. Not much is known about the general structure of such sets. One of the fundamental results is Courant's nodal domain theorem. It states that the nodal set of a $k$ th eigenfunction of the Laplacian divides a domain into at most $k$ regions in the case of the fixed membrane problem, and $k+1$ regions in the case of the free membrane problem. (Unless stated otherwise, we do not assume

Received by the editors March 19, 2004.

2000 Mathematics Subject Classification. Primary 53D10.

Key words and phrases. Nodal lines, dividing curves, contact structures, eigenfunctions of Laplacian.

This research was partially supported by NSF grant DMS-0134408.

(C)2005 American Mathematical Society 2399

Reverts to public domain 28 years from publication 
that the eigenvalues have 1-dimensional eigenspaces.) Courant's theorem implies that the first (second) eigenfunction of $\Delta_{\Sigma}$ has to divide the region into exactly two domains for the free (fixed) membrane problem. Since an eigenfunction $f$ with nonzero eigenvalue satisfies $\int_{\Sigma} f=0$, the eigenfunction always changes sign and the nodal set must therefore be nonempty.

In [23, L. E. Payne conjectured that in the case of the fixed membrane problem for bounded domains in $\mathbb{R}^{2}$, the second eigenfunction of the Laplacian cannot possess a closed nodal curve.

Conjecture 1.1 (Payne (1967)). The second eigenfunction of the Laplacian on a bounded region $\Omega$ in Euclidean $\mathbb{R}^{2}$ with the Dirichlet boundary conditions cannot have a closed curve in its nodal set.

Since 1967, Payne's conjecture has been proved to be true in the case of convex domains (see [1] and 22]). Recently, it has been proved false by T. HoffmannOstenhof and co-authors (see [16]), in the case of a non-simply connected domain (disk with slits on an inner circle removed). It is still not known, however, if Conjecture 1.1 is true for an arbitrary simply connected region in $\mathbb{R}^{2}$. In [11, P. Freitas has shown that the conjecture fails in the case of $\Omega=D^{2}$ for a nonEuclidean metric.

We consider a more global version of Payne's conjecture:

Problem 1.2. Does the first $\Delta_{\Sigma^{-}}$eigenfunction on a given closed surface $\Sigma$ of genus $\geq 1$ admit a contractible nodal curve in $\Sigma$ ?

The principal result of the paper is the construction of examples which answer this question in the affirmative.

We observe that Problem 1.2 is closely related to ideas coming from the topology of contact structures: in dimension three, these are fields of 2-d planes which are maximally nonintegrable - they are as far away from defining a foliation as possible. The topology of contact structures is extremely interesting and has been the focus of much research among topologists. In particular, much progress has been made through the elucidation of a dichotomy between the tight and overtwisted contact structures (see Section 2 for definitions).

Our principal observation is a connection between certain topological features of a contact structure - so-called dividing curves associated to a convex surface $\Sigma$ and the nodal sets for eigenfunctions of the Laplacian on $\Sigma$. These results yield a reformulation of Problem 1.2.

Problem 1.3. Given $(\Sigma, g)$, is the contact structure on $\Sigma \times \mathbb{R}$ induced by the first eigenfunction of $\Delta_{\Sigma}$ overtwisted?

These two conjectures are equivalent and provide some basis for a spectral geometry interpretation of the tight-overtwisted dichotomy in contact topology.

1.2. Outline and terminology. In Section 2, we give a short overview of contact topology and introduce the relevant definitions. The next section is devoted to the relationship between nodal sets and dividing curves in contact geometry. Section 3 contains a proof of our Main Theorem which provides counterexamples to Payne's conjecture on closed Riemannian surfaces.

Main Theorem. For an arbitrary closed compact orientable surface $\Sigma$, there always exists a smooth metric $g_{\Sigma}$ such that $\Xi(\Sigma)$ is a single embedded circle which bounds a disc in $\Sigma$. 
The technique of the proof is based on the work of J. Takahashi, 27], concerning collapsing connected sums of surfaces, which is in turn based on work of C. Anné (see [3]). As an additional result, we show the $C^{\infty}$-convergence of eigenfunctions on compact subsets of the "non-collapsing" part of the manifold.

Here, all manifolds, unless stated otherwise, are equipped with a Riemannian metric, and are compact smooth orientable of dimension either two or three. Throughout the article $C^{j}(M)$ stands for the set of $j$-differentiable functions on $M$, with $j=\infty$ smooth. Spaces $L^{2}(M), H^{j}(M)$ are customary, square integrable real functions, and are the Sobolev space of real-valued functions with at least $j$ bounded weak derivatives. The space $\Omega^{k}(M)=C^{\infty}\left(\Lambda^{k} M\right)$ is a set of smooth real-valued $k$ differential forms on $M$ making $\Omega^{*}(M)=\bigoplus_{k=0}^{n} \Omega^{k}(M)$ a graded $C^{\infty}(M)$ module over $\mathbb{R}$, where $n=\operatorname{dim}(M)$. Here we denote by $L^{2}\left(\Lambda^{k} M\right)$ and $H^{j}\left(\Lambda^{k} M\right)$, respectively, the square integrable and the Sobolev spaces of $k$-differential forms, where the measure is induced from the Riemannian metric. The Riemannian metric also induces an $L^{2}$-isometry: $*: \Omega^{k}(M) \rightarrow \Omega^{n-k}(M)$, namely the Hodge star operator. Consequently, we obtain de Rham graded complexes $\left(\Omega^{*}(M), d\right)$ and $\left(\Omega^{*}(M), \delta\right)$, where $d \equiv d^{k}: \Omega^{k}(M) \rightarrow \Omega^{k+1}(M)$ is an exterior derivative (also called a differential), and $\delta \equiv \delta^{k}: \Omega^{k+1}(M) \rightarrow \Omega^{k}(M)$ an adjoint of $d$ (also called a co-differential) given in terms of the Hodge star by $\delta^{k}=(-1)^{n k+1} * d^{n-k} *$ or equivalently as a formal adjoint of $d$,

$$
\left(d^{k} \omega, \eta\right)_{L^{2}\left(\Lambda^{k+1} M\right)}=\left(\omega, \delta^{k} \eta\right)_{L^{2}\left(\Lambda^{k} M\right)}, \quad \omega \in \Omega^{k}(M), \eta \in \Omega^{k+1}(M) .
$$

Most of the time we skip the superscripts in the notation for differentials and codifferentials, and simply write $d$ and $\delta$. The Laplacian on $k$-forms is defined by $\Delta=\delta d+d \delta$, which in the case of functions reduces to $\Delta=\delta d$ (for further reference consult [26] or 4]).

We also introduce the following notation for nodal sets. Let $\Xi(M, f)=\{x \in M$ : $f(x)=0\}$ stand for the zero set of the function $f$. In the case $f=f_{k}$, where $f_{k}$ is a $k$ th eigenfunction of $\Delta_{M}$, we write $\Xi(M, k):=\Xi\left(M, f_{k}\right)$ or $\Xi(M):=\Xi(M, 1):=$ $\Xi\left(M, f_{1}\right)$ for $k=1$.

\section{Contact structures And their Dividing Curves}

Let $M$ be a three-dimensional, closed oriented manifold. A smooth plane field $\xi$ on $M$ is called a contact structure if $\xi$ is maximally nonintegrable; that is, for any pair of vector fields $X$ and $Y$ satisfying $X_{p}, Y_{p} \in \xi_{p} \subset T_{p} M$ locally, we have $[X, Y]_{p} \notin \xi_{p}$. (This condition is exactly the opposite of the Frobenius condition for integrable subbundles.) The plane field $\xi$ can always be defined as the kernel of the differential 1-form $\alpha$ on $M$. The 1-form $\alpha$ is determined up to a multiplication by a positive real-valued function and is called a contact form. In terms of $\alpha$ the condition of non-integrability can be expressed as follows:

$$
\alpha \wedge d \alpha \neq 0 \text {. }
$$

It was proved some time ago by R. Lutz and J. Martinet (in 21]) that every closed 3-manifold admits a contact structure. Since then, there has been a significant amount of research devoted to the problem of classifying contact structures up to an isotopy of plane fields (see e.g. [9], 17], [18]). One of the fundamental results in this direction is a theorem of Y. Eliashberg [8] which divides contact structures into two classes: overtwisted and tight. 
Definition 2.1. A contact structure $\xi$ is overtwisted if and only if there exists an embedded disk $D^{2} \subset M$ such that $D$ is transverse to $\xi$ near $\partial D$ but $\partial D$ is tangent to $\xi$. Any contact structure which is not overtwisted is called tight.

In 8, Y. Eliashberg classified overtwisted contact structures in terms of the homotopy type of plane fields. On the other hand, the complete classification of tight structures still remains an open problem. In the study of this problem the concept of dividing curves for convex surfaces plays a major role [19], 17, [18.

Definition 2.2. A convex surface is a properly embedded surface $\Sigma$ in $(M, \xi)$ such that there exists a vector field $v \pitchfork \Sigma$ transverse to $\Sigma$ and preserving $\xi$ (i.e. $\mathcal{L}_{v} \xi=0$ ). The vector field $v$ is called a contact vector field. The dividing set, $\Gamma_{\Sigma}$, is the set of all points $p$ on the surface $\Sigma$, where $v_{p} \in \xi_{p}$.

The following theorem by Giroux (in [15]) characterizes the dividing set $\Gamma_{\Sigma}$.

Theorem 2.3. Let $\Sigma$ be a convex surface in $(M, \xi)$. The dividing set $\Gamma_{\Sigma}$ of $\xi$ is a set of smooth curves. Moreover, the isotopy type of $\Gamma_{\Sigma}$ is independent of choice of the contact field $v$.

A parallel theorem (also in [15]) gives a local classification result for contact structures in a tubular neighborhood of a convex surface.

Theorem 2.4. If $\Sigma \neq S^{2}$ is a convex surface for $\xi$, then $\Sigma$ has a tight neighborhood in $M$ if and only if no component of $\Gamma_{\Sigma}$ is contractible in $\Sigma$. If $\Sigma=S^{2}$, then $\Sigma$ has a tight neighborhood if and only if $\Gamma_{\Sigma}$ has only one component.

Our objective is to show that, for a special choice of a Riemannian metric, in a tubular neighborhood of $\Sigma$ the dividing set $\Gamma_{\Sigma}$ becomes the set of nodal curves for a $\Delta_{\Sigma}$-eigenfunction on $\Sigma$. In the next section we discuss a metric adaptation to contact structures.

\subsection{Adapted metrics for contact structures.}

Definition 2.5. A given metric $g$ is a contact metric for a contact form $\alpha$ if it satisfies

$$
d \alpha=* \lambda \alpha, \quad g(\alpha, \alpha) \neq 0, \quad \lambda \in C^{\infty}(M), \lambda(x) \neq 0 \quad \text { for all } x \in M,
$$

where $*$ is the Hodge star operator induced by $g$.

In [7, the authors prove that any contact form $\alpha$ admits such a metric (the definitions used there are slightly stronger).

Lemma 2.6. Any 1-form $\alpha$ satisfying condition (3) for some contact metric $g$ is a contact form.

Proof. One checks the contact condition (2). We have

$$
\alpha \wedge d \alpha=\alpha \wedge(\lambda * \alpha)=\lambda g(\alpha, \alpha) \mu, \quad \mu=* 1 .
$$

By assumptions in (3) we obtain $\alpha \wedge d \alpha \neq 0$.

Every contact metric is, in fact, fully determined by a choice of an adapted (co)frame, and can be expressed in terms of a contact form $\alpha$ and its differential $d \alpha[20$. 
2.2. Nodal sets and dividing curves of contact structures. Recall from the Introduction that the dividing set $\Gamma_{\Sigma}$ of a convex surface $\Sigma$ embedded in $(M, \xi)$ is the set of all points $p$, where the contact field $v_{p}$ belongs to contact planes $\xi_{p}$.

Lemma 2.7. Let $\Sigma$ denote a closed surface and let $\alpha$ be a contact form on $\Sigma \times$ $(-1,1)$ such that each $\Sigma \times\{t\}$ is convex with a contact field $\partial_{t}=v$ preserving $\alpha$, i.e., $\mathcal{L}_{v} \alpha=0$. Assume furthermore that $g$ is a contact metric satisfying

(i) $\lambda=$ const,

(ii) for each $t \in(-1,1)$, $v$ is orthonormal to $\Sigma \times\{t\}$ with respect to $g$.

Then the dividing set $\Gamma_{\Sigma}$ of $\alpha$ is precisely the nodal set of a $\lambda^{2}$-eigenfunction of $\Delta_{\Sigma}$ on $\left(\Sigma, g_{\Sigma}\right)$, where $g_{\Sigma}$ is the induced metric on $\Sigma$.

Proof. By assumption, we can choose a coframe $\left\{\theta_{1}, \theta_{2}, \theta_{3}\right\}$, such that $\theta_{1}=g(v, \cdot)$, and $\left\{\theta_{2}, \theta_{3}\right\} \in \Omega^{1}(\Sigma)$ is an orthonormal coframe on $\Sigma$. Denote by $\left\{X_{1}, X_{2}, X_{3}\right\}$ a dual frame $\left(v=X_{1}\right)$. We can express $\alpha$ in the coframe as follows:

$$
\alpha=f \theta_{1}+\beta, \quad \text { where } \beta=\phi_{2} \theta_{2}+\phi_{3} \theta_{3} .
$$

Therefore,

$\left.\Gamma_{\Sigma}=\left\{p \in \Sigma \times\{0\} ; v_{p} \in \xi_{p}\right\}=\left\{p \in \Sigma \times\{0\} ; v_{p}\right\lrcorner \alpha_{p}=f(p)=0\right\}=f^{-1}(0) \cap \Sigma \times\{0\}$.

Now, we show that $\beta \in \Omega^{1}(\Sigma)$. Note that the requirement $\mathcal{L}_{v} \alpha=0$ together with (3) implies

$$
\begin{aligned}
0 & \left.\left.=\mathcal{L}_{v} \alpha=v\right\lrcorner d \alpha+d f=v\right\lrcorner * \lambda \alpha+d f \\
& =\lambda v\lrcorner\left(f \theta_{2} \wedge \theta_{3}-\phi_{2} \theta_{1} \wedge \theta_{3}+\phi_{3} \theta_{1} \wedge \theta_{2}\right)+d f \\
& =-\lambda v\lrcorner\left(\theta_{1} \wedge *_{\Sigma} \beta\right)+d f
\end{aligned}
$$

where $*_{\Sigma}$ is the Hodge star operator on $\left(\Sigma, g_{\Sigma}\right)$. It follows that

$$
d f=\lambda *_{\Sigma} \beta \Rightarrow *_{\Sigma} d f=-\lambda \beta \text {. }
$$

Since $d f=\sum_{i}\left(X_{i} f\right) \theta_{i}$, we obtain the following equations for $f, \phi_{2}, \phi_{3}$ :

$$
\left\{\begin{array}{l}
X_{2} f=\lambda \phi_{3}, \\
X_{3} f=-\lambda \phi_{2}, \\
X_{1} f=0
\end{array}\right.
$$

Choosing local coordinates $(t, x, y)$, so that $v=\partial_{t}$ and $\left(\partial_{x}, \partial_{y}\right)$ are tangent to the surface $\Sigma$, we conclude that functions $f, \phi_{2}, \phi_{3}$ depend just on $(x, y)$ and $\beta \in \Omega^{1}(\Sigma)$. It follows that $d \beta=d_{\Sigma} \beta$ and $d \theta_{1}=d d t=0$, and $* \beta=-\theta_{1} \wedge\left(*_{\Sigma} \beta\right)$. As a consequence of this, and (4), we obtain

$$
* d\left(f \theta_{1}\right)=*\left(d f \wedge \theta_{1}\right)=-\lambda *\left(\theta_{1} \wedge *_{\Sigma} \beta\right)=\lambda * * \beta=\lambda \beta .
$$

Expressing condition (3) in terms of $\alpha=f \theta_{1}+\beta$, we have

$$
\begin{aligned}
\lambda \alpha=\lambda f \theta_{1}+\lambda \beta & =* d \alpha=* d\left(f \theta_{1}\right)+* d \beta=\lambda \beta+* d \beta \\
& \Rightarrow * d \beta=\lambda f \theta_{1} .
\end{aligned}
$$

It follows that

$$
*_{\Sigma} d \beta=\lambda f .
$$

Equations (41) and (6) imply that $\Delta_{\Sigma} f=\lambda^{2} f$, where $\Delta_{\Sigma}=-*_{\Sigma} d *_{\Sigma} d$. Therefore $\Gamma_{\Sigma}$ is the nodal set of $f$. 
Observe that in the frame $\left\{\theta_{i}\right\}_{i}$, the adapted metric $g$ is given by

$$
g=\sum_{i} \theta_{i}^{2}=d t^{2}+g_{\Sigma}, \quad \text { i.e. in coordinates }(t, x, y), \quad g=\left(\begin{array}{cc}
1 & 0 \\
0 & g_{\Sigma}
\end{array}\right),
$$

where $g_{\Sigma}=\theta_{2}^{2}+\theta_{3}^{2}$ is an induced metric on $\Sigma$. Hence $g=1 \oplus g_{\Sigma}$ is a product metric on $U=\Sigma \times(-1,1)$. (One can prove the above lemma directly from the decomposition of the Laplacian on $U$ in the product metric.)

Remark 2.8. If we restrict the coframe $\left\{\theta_{i}\right\}$ to $\Sigma \hookrightarrow \Sigma \times\{0\} \subset \Sigma \times(-1,1)$, the calculation in the above proof is still valid. Thus the result holds if we assume that $\theta_{1}=g(v, \cdot)$ is a part of the orthonormal coframe, and $\left.d \theta_{1}\right|_{\Sigma \times\{0\}}=0$. In other words, the metric $g$ can be only "infinitesimally" given as in (7).

For an arbitrary $\Delta_{\Sigma}$-eigenfunction $f$ on $\left(\Sigma, h_{\Sigma}\right)$, where $h_{\Sigma}$ is a smooth metric, the set of equations (5) determines a 1-form $\alpha$ in a thickening of the surface $\Sigma$. If the nodal set of $f$ does not contain singular points, then $\alpha(x) \neq 0$ for all $x$ and $\alpha$ satisfies (3) in the product metric $g=1 \oplus h_{\Sigma}$ (see [20] for the explicit calculation). Consequently, we obtain the following.

Theorem 2.9. If $\Xi(\Sigma, k)$ is the set of nonsingular nodal curve for a $k$ th eigenfunction $f$ of the Laplace operator $\Delta_{\Sigma}$ on $\left(\Sigma, h_{\Sigma}\right)$. Then there exists a contact form $\alpha$ in the thickening $\Sigma \times(-1,1)$ of $\Sigma$ such that

(i) $\Sigma$ is a convex surface for a contact structure $\xi_{\alpha}=\operatorname{ker}(\alpha)$,

(ii) $\Xi(\Sigma, k)$ is the dividing set of $\xi_{\alpha}$,

(iii) $\alpha$ is an $\lambda$-eigenform of the curl operator $* d$, where $\lambda^{2}$ is the $\Delta_{\Sigma}$-eigenvalue of $f$.

We say further that $\alpha$ is induced by $f$ in the thickening of $\Sigma$.

2.3. The topological version of Payne's conjecture. Based on results of the previous section we state the topological version of Payne's conjecture in the following question.

Problem 2.10. Is the contact structure "induced" by the first $\Delta_{\Sigma}$-eigenfunctions in the sense of Theorem 2.9, always tight in the thickening of the convex surface $\Sigma ?$

In light of Giroux's Theorem 2.4 the answer is positive if $\Sigma \simeq S^{2}$. This is a consequence of Courant's theorem, which implies that there are exactly two nodal

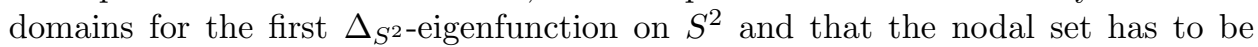
a single embedded circle. Thus $\# \Gamma_{\Sigma}=\# \Xi\left(S^{2}\right)=1$, and the associated contact structure has to be tight. In the case $\Sigma$ is an orientable surface of genus $\geq 1$, Problem 2.10 is equivalent to Problem 1.2 posed in the Introduction. In order to give a negative answer to Problem 2.10 it suffices to construct a metric on $\Sigma$ such that the first $\Delta_{\Sigma^{-}}$eigenfunction has a closed nodal curve bounding a disc. We devote the remaining part of this paper to a rigorous construction of such metrics for orientable surfaces of an arbitrary genus. Note that Theorem 2.9 does not give us any control over the eigenvalue $\lambda$, namely $\lambda$ is not necessarily the first eigenvalue of the curl operator. In [13] we address this issue, and using the Main Theorem we show existence of an overtwisted energy-minimizing eigenfield of the curl operator (which originally has been conjectured to be false in [10]). 


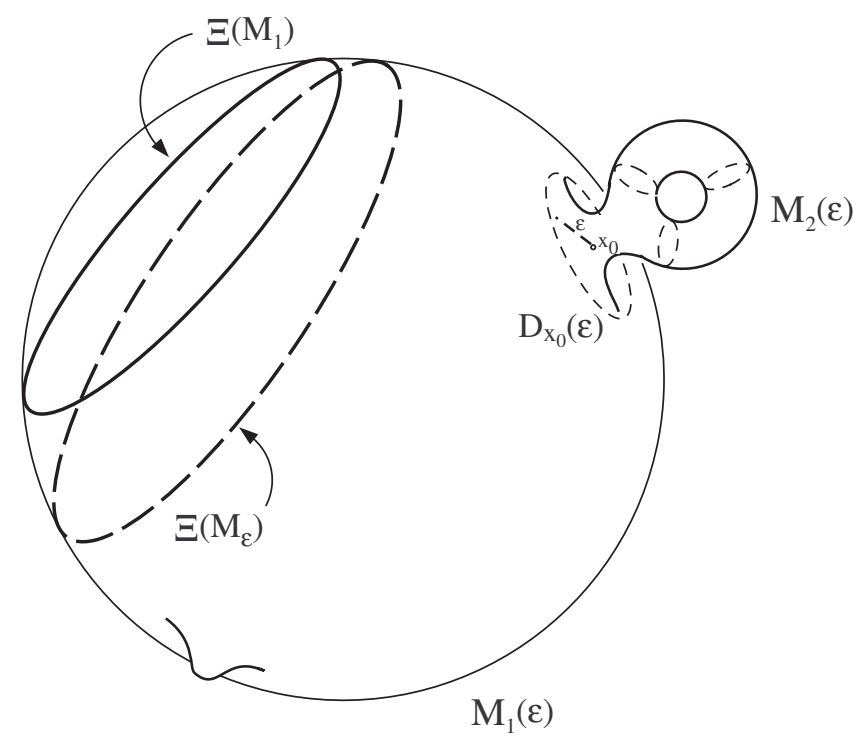

Figure 1. For small $\varepsilon$, nodal sets $\Xi\left(M_{\varepsilon}\right)$ and $\Xi\left(M_{1}\right)$ have to be "close" in $M_{\varepsilon}=\left(M_{1}(\varepsilon) \cup_{\Phi_{\varepsilon}} M_{2}(\varepsilon), g_{\varepsilon}\right)$.

\section{Closed nodal Curves for the free membrane problem}

Recall from the Introduction that the Laplace-Beltrami operator $\Delta_{M}=\delta d$ is a positive formally self-adjoint operator on any closed orientable Riemannian manifold $(M, g)$. By the standard spectral theory of formally self-adjoint operators, the $L^{2}$ - spectrum of $\Delta_{M}$ is real and countable,

$$
0=\lambda_{0}(M)<\lambda_{1}(M) \leq \lambda_{2}(M) \leq \cdots \leq \lambda_{k}(M) \leq \cdots \rightarrow \infty,
$$

and one can choose an orthonormal basis of eigenvectors $\left\{f_{i}\right\}_{i \in \mathbb{N} \cup\{0\}}$ in $L^{2}(M)$ (which are smooth by regularity),

$$
\Delta_{M} f_{k}=\lambda_{k}(M) f_{k}, \quad\left\|f_{k}\right\|_{L^{2}(M)}=1, \quad f_{k} \in C^{\infty}(M) .
$$

The main objective of this section is to prove

Main Theorem. For an arbitrary closed compact orientable surface $\Sigma$, there always exists a smooth metric $g_{\Sigma}$ such that $\Xi(\Sigma)$ is a single embedded circle which bounds a disc in $\Sigma$.

As already argued in the previous section, this statement is a straightforward corollary in the case of $\Sigma=S^{2}$. Namely, it is enough to choose a generic metric and refer to the result of K. Uhlenbeck ([28]), which states that $\Xi\left(S^{2}\right)$ has to be a one-dimensional submanifold. By Courant's Theorem $\Xi\left(S^{2}\right)$ splits $S^{2}$ into two open domains, thereby implying that $\Xi\left(S^{2}\right)$ must be a single embedded circle. If the surface is of genus $\geq 1$, we produce a desired metric by gluing via boundary circles a "big" sphere $M_{1} \simeq S^{2}$ with an $\varepsilon$-disc removed, $M_{1}(\varepsilon) \simeq S^{2} \backslash \operatorname{Int}\left(D^{2}\right) \simeq D^{2}$, to an $\varepsilon$ - "small" surface $M_{2}(\varepsilon)$, homeomorphic to $\Sigma \backslash \operatorname{Int}\left(D^{2}\right)$. The resulting manifold $M_{\varepsilon}$ is homeomorphic to $\Sigma$, and as $\varepsilon \rightarrow 0$ we show that the nodal set $\Xi\left(M_{\varepsilon}\right)$ converges to $\Xi\left(M_{1}\right)$. (See Figure 1) Thus for sufficiently small $\varepsilon=\hat{\varepsilon}, \Xi\left(M_{\hat{\varepsilon}}\right)$ has to be a closed embedded circle that belongs to $M_{1}(\hat{\varepsilon}) \simeq D^{2}$. 
3.1. Definition of $M_{\varepsilon}$. First, observe the following elementary construction. If we choose an embedded contractible 2-disc $D^{2}$ in an orientable surface $\Sigma$ and define $\Sigma^{\prime} \cong \Sigma \backslash \operatorname{Int}\left(D^{2}\right)$, then, for an arbitrary diffeomorphism $\Phi: \partial D^{2} \rightarrow \partial \Sigma^{\prime}$, we can always form a topological manifold $\Sigma_{\Phi}=D^{2} \cup_{\Phi} \Sigma^{\prime}$ by gluing $D^{2}$ back to $\Sigma^{\prime}$ via $\Phi$ (see e.g. 12]). Since $\Sigma_{\Phi}$ is homeomorphic to $\Sigma$, we can make $\Sigma_{\Phi}$ into a smooth manifold by pulling back the differential structure from $\Sigma$. All $\Sigma_{\Phi}$ obtained this way are diffeomorphic. If we equip $D^{2}$ and $\Sigma^{\prime}$ with smooth Riemannian metrics $g_{1}^{\prime}$ and $g_{2}^{\prime}$, we can define a piecewise smooth metric $g$ on $\Sigma_{\Phi}$ as follows:

$$
g= \begin{cases}g_{1}^{\prime} & \text { on } D^{2}, \\ g_{2}^{\prime} & \text { on } \Sigma^{\prime}\end{cases}
$$

Now $g$ is continuous on $\Sigma_{\Phi}$ if the gluing map $\Phi$ is an isometry. In the case $\Phi$ admits an extension to the smooth isometry of tubular neighborhoods of boundaries $\partial D^{2}$, $\partial \Sigma^{\prime}$, the metric $g$ is smooth as well.

Consider an arbitrary smooth metric on $S^{2}$ which is flat around $x_{0}$. By adding a small perturbation with support away from $x_{0}$, we can produce a generic metric $g_{1}$ on $S^{2}$ flat in a small neighborhood $U_{x_{0}}$ of the point $x_{0}$, and such that all the eigenvalues $\left\{\lambda_{k}\left(M_{1}\right)\right\}_{k}$ are simple in $g_{1}$. (Consult [28, p. 1074, for a precise definition of a metric perturbation and a rigorous proof of this fact in Theorem 8, p. 1076.) Let $M_{1}=\left(S^{2}, g_{1}\right)$ be a 2 -sphere obtained via this process. Additionally, we assume that $x_{0} \notin \Xi\left(M_{1}\right)$; otherwise we choose a different point in the flat neighborhood. Let $D_{x_{0}}^{2}(\varepsilon) \subset U_{x_{0}}$ be a geodesic disc around $x_{0}$ of radius $\varepsilon$ which is smaller than a geodesic distance between $x_{0}$ and $\Xi\left(M_{1}\right)$. Then for any $\varepsilon>0$, $M_{1}(\varepsilon)=\left(M_{1} \backslash \operatorname{Int}\left(D_{x_{0}}^{2}(\varepsilon)\right), g_{1}\right)$ is diffeomorphic to $D^{2}$. In order to obtain a metric on $\Sigma^{\prime}$, we do not make any extra assumptions, we simply choose an arbitrary smooth metric $g_{2}$ on $\Sigma$, flat around a given point $x_{1}$, and a geodesic disc $D_{x_{1}}^{2}(r)$ of radius $r$ which belongs to the flat neighborhood. Clearly, $\Sigma \backslash D_{x_{1}}^{2}(r)$ is diffeomorphic to $\Sigma^{\prime}$, and since the metric $g_{2}$ can be always rescaled, we may assume that $r=1$. Define $M_{2}(\varepsilon)=\left(\Sigma^{\prime}, \varepsilon^{2} g_{2}\right)$.

For any $\varepsilon>0$, choose local coordinates $(x, y)$ such that the geodesic disc $D_{x_{0}}^{2}(\varepsilon)$ is an $\varepsilon$ disc on $\left(\mathbb{R}^{2}, d^{2} s\right)$ and $D_{x_{1}}^{2}(\varepsilon)$ is a unit disc on $\left(\mathbb{R}^{2}, \varepsilon^{2} d^{2} s\right)$, where $d s^{2}=d x^{2}+d y^{2}$. Observe that the boundaries $\partial M_{1}(\varepsilon), \partial M_{2}(\varepsilon)$ can be glued via an isometry $\Phi_{\varepsilon}$ of $\left(\mathbb{R}^{2}, d^{2} s\right)$ and $\left(\mathbb{R}^{2}, \varepsilon^{2} d^{2} s\right)$ restricted to a circle of radius $\varepsilon$ in $\left(\mathbb{R}^{2}, d^{2} s\right)$. (The isometry $\Phi_{\varepsilon}$ can be defined as $\Phi_{\varepsilon}: x \rightarrow(1 / \varepsilon) x$.) By the discussion in the first paragraph of this section we can form a smooth manifold $M=M_{1}(\varepsilon / 4) \cup_{\Phi_{\varepsilon / 4}} M_{2}(\varepsilon / 4)$ (the factor $\varepsilon / 4$ is chosen for convenience). We define a piecewise smooth continuous metric on $M$ as before (see also [27])

$$
\widetilde{g}_{\varepsilon}= \begin{cases}g_{1} & \text { on } D^{2} \\ (\varepsilon / 4)^{2} g_{2} & \text { on } \Sigma^{\prime}\end{cases}
$$

According to [27] the following convergence of eigenvalues holds.

Theorem 3.1. For all $k=0,1, \ldots$, we have

$$
\lim _{\varepsilon \rightarrow 0} \lambda_{k}\left(M, \widetilde{g}_{\varepsilon}\right)=\lambda_{k}\left(M_{1}, g_{1}\right) \text {. }
$$

Remark 3.2. Our main objective is to prove convergence of nodal sets in $M$ to the nodal curve of $M_{1}$. Since the metric $\widetilde{g}_{\varepsilon}$ is not smooth, and we would like to show a smooth counterexample to our version of Payne's conjecture, we need to perturb $\widetilde{g}_{\varepsilon}$ in a suitable way. 
For piecewise smooth metrics, eigenvalues of the Laplacian "vary" continuously with respect to the $C^{0}$-topology (see [5, p. 162). Therefore, for a given $\varepsilon>0$ we can perturb the metric $\widetilde{g}_{\varepsilon}$ to a smooth metric $g_{\varepsilon}$ so that eigenvalues are arbitrarily "close". (See Theorem 1.2 in [27].) By Theorem 8 in 28] and results of [5], we may assume that the support of the perturbation is contained in the complement $M_{1}(\varepsilon / 2)^{c}$. Denote $\left(M, g_{\varepsilon}\right)$ by $M_{\varepsilon}$. Consequently, we can have a family of metrics $\left\{g_{\varepsilon}\right\}_{\varepsilon}$, satisfying the following requirements:

(i) $g_{\varepsilon}$ are smooth and converge to $\widetilde{g}_{\varepsilon}$ in the $C^{0}$-topology of $M$.

(ii) $\left.g_{\varepsilon}\right|_{M_{1}(\varepsilon / 2)}=g_{1}$.

(iii) $\lambda_{k}\left(M_{\varepsilon}\right)$ are all simple eigenvalues, and nodal sets $\Xi\left(M_{\varepsilon}\right)$ are embedded circles.

(iv) $\lim _{\varepsilon \rightarrow 0} \lambda_{k}\left(M_{\varepsilon}\right)=\lambda_{k}\left(M_{1}\right)$.

We summarize our notation below:

- $M=M_{1}(\varepsilon / 4) \cup_{\Phi_{\varepsilon / 4}} M_{2}(\varepsilon / 4)$,

- $M_{1}=\left(S^{2}, g_{1}\right), M_{1}(\varepsilon)=\left(M_{1} \backslash D_{x_{0}}^{2}(\varepsilon), g_{1}\right)$,

- $M_{2}(\varepsilon)=\left(\Sigma \backslash D_{x_{1}}^{2}(1), \varepsilon^{2} g_{2}\right)$,

- $M_{\varepsilon}=\left(M, g_{\varepsilon}\right)$.

If we must specify a different metric on a manifold, we write e.g. $\left(M_{2}(\varepsilon), \hat{g}\right)$.

3.2. $C^{\infty}$-convergence of eigenfunctions. Comparing nodal sets $\Xi\left(M_{\varepsilon}\right)$ and $\Xi\left(M_{1}\right)$ can be a little bit subtle. Note that for each $\varepsilon>0, M_{\varepsilon}$ is diffeomorphic to $\Sigma$ and $\left\{g_{\varepsilon}\right\}_{\varepsilon}$ is a family of metrics on $\Sigma$. In the limit $\varepsilon=0$, the metric $g_{\varepsilon}$ degenerates on $M_{2}(1)$, and $M_{0}=\left(\Sigma, g_{0}\right)$ is not homeomorphic to $M_{1}=\left(S^{2}, g_{1}\right)$. Rather, it inherits topology that is pulled back from $M_{1}$ under the quotient map, $\pi: \Sigma \rightarrow \Sigma / M_{2}(1) \simeq S^{2}$. Thus we really have no control over what happens to the nodal set in the "shrinking" part $M_{2}(\varepsilon)$ of the manifold $M_{\varepsilon}$; technically, we cannot compare eigenfunctions on $M_{1}$ to eigenfunctions $f_{k}^{\varepsilon} \in C^{\infty}\left(M_{\varepsilon}\right)$ on $M_{\varepsilon}$. We must instead restrict them to the common domain $M_{1}\left(\varepsilon_{0}\right)$ for a given $\varepsilon_{0}>0$. In order to prove the convergence of nodal sets as $\varepsilon \rightarrow 0$, we must show uniform convergence of eigenfunctions $f_{k}^{\varepsilon}$ restricted to $M_{1}\left(\varepsilon_{0}\right)$. In this section, we show that for any sequence $\left\{\varepsilon_{j}\right\}_{j} ; \varepsilon_{j} \rightarrow 0,\left\{\left.f_{k}^{\varepsilon_{j}}\right|_{M_{1}\left(\varepsilon_{0}\right)}\right\}_{\varepsilon_{j}}$ converges to $\left.f_{k}\right|_{M_{1}\left(\varepsilon_{0}\right)} \in C^{\infty}\left(M_{1}\left(\varepsilon_{0}\right)\right)$ in the $C^{\infty}$-topology. For this purpose we need suitable extensions of $\left.f_{k}^{\varepsilon}\right|_{M_{1}(\varepsilon)}$ to the entire $M_{1}$. In the proof we use the following extension lemma.

Lemma 3.3. Let $g$ be an arbitrary smooth metric on $M_{1}$. Given $u \in C^{\infty}\left(M_{1}(\varepsilon)\right) \cap$ $C^{0}\left(\overline{M_{1}(\varepsilon)}\right)$, there exists a function $\bar{u} \in H^{1}\left(M_{1}, g\right)$, which is an extension of $u$, i.e. $\left.\bar{u}\right|_{M_{1}(\varepsilon)}=u$ such that

$$
\|\bar{u}\|_{H^{1}\left(M_{1}, g\right)} \leq C\|u\|_{H^{1}\left(M_{1}(\varepsilon), g\right)},
$$

and $C$ is independent of $\varepsilon$. For $l>1$ and $u \in C^{\infty}\left(M_{1}(\varepsilon / 2)\right) \cap C^{0}\left(\overline{M_{1}(\varepsilon / 2)}\right)$, we can find an extension $\bar{u} \in C^{\infty}\left(M_{1}\right)$ such that $\left.\bar{u}\right|_{M_{1}(\varepsilon)}=u$ and

$$
\begin{aligned}
\|\bar{u}\|_{H^{l}\left(M_{1}, g\right)} & \leq C_{l, \varepsilon}^{\prime}\|u\|_{H^{l}\left(M_{l}(\varepsilon / 2), g\right)}, \\
\left\|\Delta_{M_{1}} \bar{u}\right\|_{H^{l-2}\left(M_{1}, g\right)} & \leq C_{l, \varepsilon}^{\prime \prime}\left(\|u\|_{H^{l-2}\left(M_{l}(\varepsilon / 2), g\right)}+\|d u\|_{H^{l-2}\left(\Lambda^{1} M_{l}(\varepsilon / 2), g\right)}\right. \\
& \left.+\left\|\Delta_{M_{1}} u\right\|_{H^{l-2}\left(M_{l}(\varepsilon / 2), g\right)}\right),
\end{aligned}
$$

where constants $C_{l, \varepsilon}^{\prime \prime}, C_{l, \varepsilon}^{\prime}$ depend on $l$ and $\varepsilon$. 
Proof. The proof for $l=1$ and $\operatorname{dim}=2$ is given in 24], p. 40, where the authors show that for the unique harmonic extension the constant $C$ is independent of $\varepsilon$. For the proof in the case $l>1$ we follow the standard extension argument; see [14. We define $\bar{u}=u \rho$, where $\rho \in C^{\infty}\left(M_{1}\right)$ is a "bump" function such that $\left.\rho\right|_{M_{1}(\varepsilon)}=1$ and $\operatorname{supp}(\rho) \subset M_{1}(\varepsilon / 2)$. Inequality (11) follows immediately, and (12) is a consequence of the triangle inequality and the following product formula:

$$
\Delta_{M_{1}}(\bar{u})=\Delta_{M_{1}}(u \rho)=u \Delta_{M_{1}} \rho-2 g(\nabla u, \nabla \rho)+\rho \Delta_{M_{1}} u,
$$

which holds pointwise (see e.g. [26]).

Theorem 3.4. For each $k$, and an arbitrary $j>2$, the following $C^{j}$-convergence of eigenfunctions $f_{k}^{\varepsilon} \in C^{\infty}\left(M_{\varepsilon}\right),\left\|f_{k}^{\varepsilon}\right\|_{L^{2}\left(M_{\varepsilon}\right)}=1$ holds:

$$
\lim _{\varepsilon \rightarrow 0} f_{k}^{\varepsilon}=f_{k} \quad \text { on compact subsets of } \quad M_{1} \backslash\left\{x_{0}\right\},
$$

where $f_{k} \in C^{\infty}\left(M_{1}\right)$ is a kth $\Delta_{M_{1}}$-eigenfunction on $M_{1}$.

The above theorem leads to an immediate corollary.

Corollary 3.5. Convergence (14) holds on compact subsets of $M_{1} \backslash\left\{x_{0}\right\}$ in the $C^{\infty}$-topology of $M_{1}$.

Proof of Theorem 3.4. For a given eigenfunction $f_{k}^{\varepsilon}$ on $M_{\varepsilon}$, we introduce the following notation; $f_{k}^{\varepsilon}=\left(f_{k}^{1, \varepsilon}, f_{k}^{2, \varepsilon}\right)$, where $f_{k}^{1, \varepsilon}=\left.f_{k}^{\varepsilon}\right|_{M_{1}(\varepsilon)}, f_{k}^{2, \varepsilon}=\left.f_{k}^{\varepsilon}\right|_{M_{2}(\varepsilon)}$. For convenience we also assume $\varepsilon<1$. First we prove that there is a family of extensions $\left\{\hat{f}_{k}^{1, \varepsilon}\right\}_{\varepsilon},\left.\hat{f}_{k}^{1, \varepsilon}\right|_{M_{1}(\varepsilon)}=f_{k}^{1, \varepsilon}$, convergent in $L^{2}\left(M_{1}\right)$ to $f_{k}$. (The argument is essentially the same as in [27, p. 206.)

Choose $\hat{f}_{k}^{1, \varepsilon}$ to be the $H^{1}$-extensions of $f_{k}^{1, \varepsilon} \in C^{\infty}\left(M_{1}(\varepsilon)\right)$ given by Lemma 3.3. We have the following:

$$
\left\|\hat{f}_{k}^{1, \varepsilon}\right\|_{H^{1}\left(M_{1}\right)} \leq C\left\|f_{k}^{1, \varepsilon}\right\|_{H^{1}\left(M(\varepsilon), g_{1}\right)},
$$

where $C$ is independent of $\varepsilon$. From (15) we obtain

$$
\begin{aligned}
\left\|\hat{f}_{k}^{1, \varepsilon}\right\|_{H^{1}\left(M_{1}\right)} & \leq C\left\|f_{k}^{\varepsilon}\right\|_{H^{1}\left(M_{1}(\varepsilon), g_{1}\right)} \stackrel{(1)}{\leq} C\left(\left\|f_{k}^{\varepsilon}\right\|_{L^{2}\left(M_{\varepsilon}\right)}+\left\|d f_{k}^{\varepsilon}\right\|_{L^{2}\left(\Lambda^{1} M_{\varepsilon}\right)}\right) \\
& =C\left(1+\left(\Delta_{M_{\varepsilon}} f_{k}^{\varepsilon}, f_{k}^{\varepsilon}\right)_{L^{2}\left(M_{\varepsilon}\right)}\right) \stackrel{(2)}{=} C\left(1+\lambda_{k}^{\frac{1}{2}}\left(M_{1}\right)+\eta_{\varepsilon}\right),
\end{aligned}
$$

where $\eta_{\varepsilon} \rightarrow 0$ as $\varepsilon \rightarrow 0$. Inequality (1) follows from the definition of the $H^{1}$-norm, assumption (ii) on $g_{\varepsilon}$ and the fact that $M_{1}(\varepsilon) \subset M_{\varepsilon}$; the equality (2) is a consequence of assumption (iv) on $g_{\varepsilon}$. We conclude that the family $\left\{\hat{f}_{k}^{1, \varepsilon}\right\}_{\varepsilon}$ is bounded in $H^{1}\left(M_{1}\right)$, thus any sequence in the family contains a weakly convergent subsequence in $H^{1}\left(M_{1}\right)$. By Rellich's Theorem, the inclusion $H^{1}\left(M_{1}\right) \hookrightarrow L^{2}\left(M_{1}\right)$ is compact, thus any sequence in the family $\left\{\hat{f}_{k}^{1, \varepsilon}\right\}_{\varepsilon}$ contains a strongly convergent subsequence $\left\{\hat{f}_{k}^{1, \varepsilon_{i}}\right\}_{\varepsilon_{i}}$ in $L^{2}\left(M_{1}\right)$. Denote a limit of the subsequence by $\hat{f}_{k} \in H^{1}\left(M_{1}\right)$. We wish to show that $\hat{f}_{k}$ is a smooth classical solution to $\Delta_{M_{1}} u=\lambda_{k}\left(M_{1}\right) u$.

Let $B: H^{1}\left(M_{1}\right) \times H^{1}\left(M_{1}\right) \rightarrow \mathbb{R}$ be the bilinear form associated to the Laplacian $\Delta_{M_{1}}$. Recall that for smooth functions $u$ and $w$,

$$
B(u, w)=(\delta d u, w)_{L^{2}\left(M_{1}\right)}=(d u, d w)_{L^{2}\left(\Lambda^{1} M_{1}\right)} .
$$


The last equality extends the definition of $B$ to $H^{1}\left(M_{1}\right)$. Letting $v \in C_{c}^{\infty}\left(M_{1} \backslash\left\{x_{0}\right\}\right)$ be a test function,

$$
\begin{aligned}
B\left(\hat{f}_{k}, v\right) & =\int_{M_{1}}\left\langle d \hat{f}_{k}, d v\right\rangle_{g_{1}} d g_{1} \stackrel{(1)}{=} \lim _{i \rightarrow \infty} \int_{M_{1}\left(\varepsilon_{i}\right)}\left\langle d f_{k}^{1, \varepsilon_{i}}, d v\right\rangle_{g_{1}} d g_{1} \\
& =\lim _{i \rightarrow \infty} \int_{M_{1}\left(\varepsilon_{i}\right)}\left\langle d f_{k}^{1, \varepsilon_{i}}, d v\right\rangle_{g_{1}} d g_{1}+\int_{M_{2}\left(\varepsilon_{i}\right)}\left\langle d f_{k}^{2, \varepsilon_{i}}, 0\right\rangle_{g_{\varepsilon_{i}}} d g_{\varepsilon_{i}} \\
& \stackrel{(2)}{=} \lim _{i \rightarrow \infty}\left(\Delta_{M_{\varepsilon}}\left(f_{k}^{1, \varepsilon_{i}}, f_{k}^{2, \varepsilon_{i}}\right),(v, 0)\right)_{L^{2}\left(M_{\varepsilon_{i}}\right)} \\
& =\lim _{i \rightarrow \infty} \lambda_{k}\left(M_{\varepsilon_{i}}\right)\left(\left(f_{k}^{1, \varepsilon_{i}}, f_{k}^{2, \varepsilon_{i}}\right),(v, 0)\right)_{L^{2}\left(M_{\varepsilon_{i}}\right)} \\
& \stackrel{(3)}{=} \lambda_{k}\left(M_{1}\right) \lim _{i \rightarrow \infty} \int_{M_{1}\left(\varepsilon_{i}\right)} \hat{f}_{k} v d g_{1}=\lambda_{k}\left(M_{1}\right)\left(\hat{f}_{k}, v\right)_{L^{2}\left(M_{1}\right)} .
\end{aligned}
$$

Equality (1) follows from the $H^{1}$-weak convergence of extensions $\hat{f}_{k}^{1, \varepsilon}$ and $\left.\hat{f}_{k}^{1, \varepsilon}\right|_{M_{1}(\varepsilon)}$ $=f_{k}^{1, \varepsilon}$. Equalities (2) and (3) follow from the assumptions (ii) and (iv) on $g_{\varepsilon_{i}}$. From the density of $C_{c}^{\infty}\left(M_{1} \backslash\left\{x_{0}\right\}\right)$ in $H^{1}\left(M_{1}, g_{1}\right)$, which holds in dimensions $\geq 2$ (see [2]), the equality $B\left(\hat{f}_{k}, v\right)=\lambda_{k}\left(M_{1}\right)\left(\hat{f}_{k}, v\right)_{L^{2}\left(M_{1}\right)}$ is valid for any $v \in H^{1}\left(M_{1}\right)$. Consequently, $\hat{f}_{k}$ is a weak solution to $\Delta_{M_{1}} u=\lambda_{k}\left(M_{1}\right) u$, and by the regularity of weak solutions we conclude that $\hat{f}_{k}$ is a smooth classical solution. Since all the eigenvalues $\lambda_{k}\left(M_{1}\right)$ are simple in $g_{1}$ and

$$
\begin{aligned}
1=\left\|f_{k}\right\|_{L^{2}\left(M_{1}, g_{1}\right)} & =\lim _{i \rightarrow \infty}\left\|f_{k}\right\|_{L^{2}\left(M_{1}\left(\varepsilon_{i}\right), g_{1}\right)} \\
& =\lim _{i \rightarrow \infty}\left\|\hat{f}_{k}^{1, \varepsilon_{i}}\right\|_{L^{2}\left(M_{1}\left(\varepsilon_{i}\right), g_{1}\right)}=\left\|\hat{f}_{k}\right\|_{L^{2}\left(M_{1}, g_{1}\right)},
\end{aligned}
$$

we conclude that $\hat{f}_{k}=f_{k}$.

In the next step, we argue $C^{j}$-convergence of $f_{k}^{1, \varepsilon_{i}}$ on compact subsets of $M_{1} \backslash$ $\left\{x_{0}\right\}$.

Choose $\varepsilon_{0}$ such that $M_{1}\left(\varepsilon_{0}\right)$ contains a given compact subset and let $l>j+$ $\frac{m}{2}+1=j+2$, where $m=2$ is the dimension of $M_{1}$ (we assume $j>2$ for convenience). Letting $\varepsilon \leq \varepsilon_{0}$, we apply Lemma 3.3 and consider a family of $H^{l}$ extensions $\bar{f}_{k}^{1, \varepsilon} \in H^{l}\left(M_{1}\right)$ for $f_{k}^{1, \varepsilon} \in C^{\infty}\left(M_{1}\left(\varepsilon_{0} / 2\right)\right)$. Applying Garding's inequality ([25], p. 76) for differential forms with a constant $D_{l}$ we have

$$
\begin{aligned}
\left\|\bar{f}_{k}^{1, \varepsilon}\right\|_{H^{l}\left(M_{1}\right)} & \leq D_{l}\left(\left\|\bar{f}_{k}^{1, \varepsilon}\right\|_{H^{l-1}\left(M_{1}\right)}+\left\|(d+\delta) \bar{f}_{k}^{1, \varepsilon}\right\|_{H^{l-1}\left(\Lambda^{*} M_{1}\right)}\right) \\
& =K_{l-1}\left(\left\|\bar{f}_{k}^{1, \varepsilon}\right\|_{H^{l-1}\left(M_{1}\right)}+\left\|d \bar{f}_{k}^{1, \varepsilon}\right\|_{H^{l-1}\left(\Lambda^{1} M_{1}\right)}\right)
\end{aligned}
$$

(where $K_{l-1}=D_{l}$ ). Here $d+\delta$ is the Dirac operator (i.e. $(d+\delta)^{2}=\Delta$ ) acting on forms of mixed degree. Applying Garding's inequality again to each term of (17) 
and setting $D_{l, l-1}=D_{l} D_{l-1}$ results in

$$
\begin{aligned}
(\text { rhs of (17) }) \leq & D_{l, l-1}\left(\left\|\bar{f}_{k}^{1, \varepsilon}\right\|_{H^{l-2}\left(M_{1}\right)}+2\left\|d \bar{f}_{k}^{1, \varepsilon}\right\|_{H^{l-2}\left(\Lambda^{1} M_{1}\right)}\right. \\
& \left.+\left\|\Delta_{M_{1}} \bar{f}_{k}^{1, \varepsilon}\right\|_{H^{l-2}\left(M_{1}\right)}\right) \\
& \stackrel{(1)}{\leq} D_{l, l-1} C_{l, \varepsilon_{0}}^{\prime \prime}\left(\left\|\bar{f}_{k}^{1, \varepsilon}\right\|_{H^{l-2}\left(M_{1}\right)}+2\left\|d \bar{f}_{k}^{1, \varepsilon}\right\|_{H^{l-2}\left(\Lambda^{1} M_{1}\right)}\right. \\
& \left.\quad\left\|\Delta_{M_{\varepsilon}} f_{k}^{\varepsilon}\right\|_{H^{l-2}\left(M_{1}\left(\varepsilon_{0} / 2\right), g_{\varepsilon}\right)}\right) \\
& \stackrel{(2)}{\leq} 2 D_{l, l-1} C_{l-2, \varepsilon_{0}}^{\prime \prime}\left(1+\lambda_{k}\left(M_{\varepsilon}\right)\right)\left(\left\|\bar{f}_{k}^{1, \varepsilon}\right\|_{H^{l-2}\left(M_{1}\right)}+\left\|d \bar{f}_{k}^{1, \varepsilon}\right\|_{H^{l-2}\left(\Lambda^{1} M_{1}\right)}\right) \\
& \stackrel{(3)}{\leq} K_{l-2}\left(\left\|\bar{f}_{k}^{1, \varepsilon}\right\|_{H^{l-2}\left(M_{1}\right)}+\left\|d \bar{f}_{k}^{1, \varepsilon}\right\|_{H^{l-2}\left(\Lambda^{1} M_{1}\right)}\right) .
\end{aligned}
$$

Inequality (1) is a consequence of (12), whereas (2) follows from the fact that $f_{k}^{\varepsilon}$ is a $k$ th eigenfunction of the Laplacian on $M_{\varepsilon}$. In (3) we set

$$
K_{l-2}>2 D_{l, l-1} C_{l-2, \varepsilon_{0}}^{\prime \prime}\left(1+\lambda_{k}\left(M_{\varepsilon}\right)\right)
$$

due to the requirement (iv) on $g_{\varepsilon}$. Repeating the above steps finitely many times leads to

$$
\begin{aligned}
\left\|\bar{f}_{k}^{1, \varepsilon}\right\|_{H^{l}\left(M_{1}\right)} & \leq K_{0}\left(\left\|\bar{f}_{k}^{1, \varepsilon}\right\|_{L^{2}\left(M_{1}\right)}+\left\|d \bar{f}_{k}^{1, \varepsilon}\right\|_{L^{2}\left(\Lambda^{1} M_{1}\right)}\right)=K_{0}\left\|\bar{f}_{k}^{1, \varepsilon}\right\|_{H^{1}\left(M_{1}\right)} \\
& \leq K_{0} C_{1, \varepsilon_{0}}^{\prime}\left(\left\|f_{k}^{1, \varepsilon}\right\|_{L^{2}\left(M_{1}\left(\varepsilon_{0} / 2\right), g_{1}\right)}+\left\|d f_{k}^{1, \varepsilon}\right\|_{L^{2}\left(\Lambda^{1} M_{1}\left(\varepsilon_{0} / 2\right), g_{1}\right)}\right) \\
& \leq K_{0} C_{1, \varepsilon_{0}}^{\prime}\left(1+\left(\Delta_{M_{\varepsilon}} f_{k}^{\varepsilon}, f_{k}^{\varepsilon}\right)_{L^{2}\left(M_{\varepsilon}\right)}^{\frac{1}{2}}\right) \leq K_{0} C_{1, \varepsilon_{0}}^{\prime}\left(1+\lambda_{k}^{\frac{1}{2}}\left(M_{1}\right)+\eta_{\varepsilon}^{\prime}\right),
\end{aligned}
$$

where $\eta_{\varepsilon}^{\prime} \rightarrow 0$ as $\varepsilon \rightarrow 0$ and we applied Lemma 3.3 in the second inequality. Consequently, the family $\left\{\bar{f}_{k}^{1, \varepsilon}\right\}_{\varepsilon}$ is bounded in $H^{l}\left(M_{1}\right)$. By Rellich's Theorem we have a compact inclusion $H^{l}\left(M_{1}\right) \hookrightarrow H^{l-1}\left(M_{1}\right)$, and by the Sobolev embedding theorem a bounded inclusion $H^{l-1}\left(M_{1}\right) \hookrightarrow C^{j}\left(M_{1}\right)$. Composition of these two gives us a compact inclusion $H^{l}\left(M_{1}\right) \hookrightarrow C^{j}\left(M_{1}\right)$. As a result, there exists a subsequence $\left\{\bar{f}_{k}^{1, \varepsilon_{i}}\right\}_{i}$ of any sequence in $\left\{\bar{f}_{k}^{1, \varepsilon}\right\}_{\varepsilon}$, convergent in the $C^{j}$ topology of $M_{1}$. Denote a limit of this subsequence by $\bar{f}_{k} \in C^{j}\left(M_{1}\right)$.

Since $\left.\hat{f}_{k}^{1, \varepsilon}\right|_{M_{1}\left(\varepsilon_{0}\right)}=\left.f_{k}^{1, \varepsilon}\right|_{M_{1}\left(\varepsilon_{0}\right)}=\left.\bar{f}_{k}^{1, \varepsilon}\right|_{M_{1}\left(\varepsilon_{0}\right)}$ for any $\varepsilon<\varepsilon_{0}$, the $L^{2}$-limits $\hat{f}_{k}$, $\bar{f}_{k}$ have to agree on $M_{1}\left(\varepsilon_{0}\right)$. Equality (14) holds, since for any sequence $\left\{\varepsilon_{i}\right\}_{i}$ converging to zero, $\left\{\left.f_{k}^{1, \varepsilon_{i}}\right|_{M_{1}\left(\varepsilon_{0}\right)}\right\}$ contains a convergent subsequence with a common limit.

3.3. Proof of the Main Theorem. We assume that the nodal curve $\Xi\left(M_{1}\right)$ is at a geodesic distance $d>\varepsilon_{0}$ from the gluing disc $D_{\varepsilon} \subset D_{\varepsilon_{0}}$. The nodal sets $\Xi\left(M_{1}\right)$ and $\Xi\left(M_{\varepsilon}\right)$ can be compared only on the common subset $M_{1}\left(\varepsilon_{0}\right)$, and since we just proved the convergence in $C^{\infty}\left(M_{1}\left(\varepsilon_{0}\right)\right)$ of $\left.\left.f_{1}^{\varepsilon_{i}}\right|_{M_{1}\left(\varepsilon_{0}\right)} \rightarrow f_{1}\right|_{M_{1}\left(\varepsilon_{0}\right)}$ for any sequence $\varepsilon_{i} \rightarrow 0$, the first impression is that we have no control over nodal sets in the "shrinking" part $M_{2}(\varepsilon)$ of the manifold $M_{\varepsilon}$. What we really have to show is that for some $\varepsilon>0$ the nodal set $\Xi\left(M_{\varepsilon}\right)$ belongs entirely to the common domain $M_{1}\left(\varepsilon_{0}\right)$. This would imply that $\Xi\left(M_{\varepsilon}\right)$ is an embedded contractible circle, since $M_{1}\left(\varepsilon_{0}\right)$ is itself contractible. First of all note the following pointwise convergence of nodal sets.

Lemma 3.6. Consider a sequence of points $\left\{x_{i}\right\}_{i}$ such that for each $i, x_{i} \in$ $\Xi\left(M_{\varepsilon_{i}}\right) \cap M_{1}\left(\varepsilon_{0}\right)$. If the limit $x$ of $\left\{x_{i}\right\}_{i}$ exists, then $x \in \Xi\left(M_{1}\right)$. 
Proof. Applying the convergence $\left.\left.f_{1}^{\varepsilon_{i}}\right|_{M_{1}\left(\varepsilon_{0}\right)} \rightarrow f_{1}\right|_{M_{1}\left(\varepsilon_{0}\right)}$ in $C^{0}\left(M_{1}\left(\varepsilon_{0}\right)\right)$ we obtain

$$
\left|f_{1}\left(x_{i}\right)\right|=\left|f_{1}\left(x_{i}\right)-f_{1}^{1, \varepsilon_{i}}\left(x_{i}\right)\right| \leq\left\|f_{1}-f_{1}^{1, \varepsilon_{i}}\right\|_{C^{0}\left(M_{1}\left(\varepsilon_{0}\right)\right)} \stackrel{i \rightarrow \infty}{\longrightarrow} 0 .
$$

By continuity of $f_{1}$ and the assumption $x_{i} \rightarrow x \in M_{1}\left(\varepsilon_{0}\right)$, we have $0=\lim _{i \rightarrow \infty} f_{1}\left(x_{i}\right)$ $=f_{1}(x)$, and we conclude that $x \in \Xi\left(M_{1}\right)$.

In order to finish the proof of the Main Theorem we need only show the following.

Claim 3.7. The nodal curves $\Xi\left(M_{\varepsilon_{i}}\right)$ are eventually in $M_{1}\left(\varepsilon_{0}\right)$, i.e. we can find an index $n$ such that for all $i>n, \Xi\left(M_{\varepsilon_{i}}\right) \subset M_{1}\left(\varepsilon_{0}\right)$. Additionally for $i>n$, each $\Xi\left(M_{\varepsilon_{i}}\right)$ is a single embedded circle.

We consider two cases: either there exists an infinite sequence of points $\left\{x_{i}\right\}_{i} \subset$ $\Xi\left(M_{\varepsilon_{i}}\right) \cap M_{1}\left(\varepsilon_{0}\right)\left(\right.$ case $\left.1^{\circ}\right)$, or not $\left(\right.$ case $\left.2^{\circ}\right)$.

Proof of the claim in case $1^{\circ}$. By compactness of $M_{1}\left(\varepsilon_{0}\right)$, we can choose a convergent subsequence of $\left\{x_{i}\right\}_{i}$. We denote the subsequence by $\left\{\hat{x}_{j}\right\}_{j}$ and its limit by $\hat{x}$. According to Lemma 3.6, $\hat{x} \in \Xi\left(M_{1}\right) \subset M_{1}\left(\varepsilon_{0}\right)$. By assumption (iii) in Section 3.1, for each $j$ we have an embedding $\phi_{j}: S^{1} \hookrightarrow M_{\varepsilon_{j}}$ such that for some $\theta_{j} \in S^{1}, \phi_{j}\left(\theta_{j}\right)=\hat{x}_{j}$. If $\varepsilon_{j}$ is sufficiently small, all elements of $\hat{x}_{j}$ belong to a geodesic ball $D_{\hat{x}}(\hat{r}) \subset M_{1}\left(\varepsilon_{0}\right)$ around $\hat{x}$ of arbitrarily small radius $\hat{r}$. To prove the claim, we reason by contradiction. Suppose that there exists a subsequence $\{m\}$ of $\{j\}$ such that $\phi_{m}\left(S^{1}\right) \nsubseteq M_{1}\left(\varepsilon_{0}\right)$. Then for each $m$ there is a point $\gamma_{m}$ where $\phi_{m}\left(\gamma_{m}\right) \notin M_{1}\left(\varepsilon_{0}\right)$. Consequently, $\phi_{m}:\left(\theta_{m}, \gamma_{m}\right) \hookrightarrow M_{\varepsilon_{m}}$ is an oriented path joining two different points, one in $M_{1}\left(\varepsilon_{0}\right)$, the other in $M_{\varepsilon_{m}} \backslash M_{1}\left(\varepsilon_{0}\right)$. Clearly, the path $\left.\phi_{m}\right|_{\left(\theta_{m}, \gamma_{m}\right)}$ has to intersect $\partial M_{1}\left(\varepsilon_{0}\right)$. Choose a convergent subsequence of intersection points $y_{m} \in \partial M_{1}\left(\varepsilon_{0}\right)$. Its limit $y \in \partial M_{1}\left(\varepsilon_{0}\right)$ belongs to $\Xi\left(M_{1}\right)$ by Lemma 3.6. As a consequence, the intersection $\Xi\left(M_{1}\right) \cap \partial M_{1}\left(\varepsilon_{0}\right)$ would have to be nonempty, which contradicts the choice of $\varepsilon_{0}$ (i.e. the nodal set $\Xi\left(M_{1}\right)$ is supposed to be at some geodesic distance $d>\varepsilon_{0}$ from the boundary of the gluing disc). Therefore, there exists an index $n$ such that $\phi_{j}\left(S^{1}\right) \subset M_{1}\left(\varepsilon_{0}\right)$ for all $j>n$. The image of $S^{1}$ under $\phi_{j}$ is a closed embedded curve in $M_{1}\left(\varepsilon_{0}\right) \simeq D^{2}$ which splits $M_{\varepsilon_{j}}$ into two domains, where the eigenfunction is either strictly positive or negative (a $\Delta_{\Sigma^{-}}$-eigenfunction cannot have a zero, so there is no change of sign in the neighborhood). By Courant's Theorem, the first eigenfunction must have exactly two nodal domains, hence $\phi_{j}\left(S^{1}\right)=\Xi\left(M_{\varepsilon_{j}}\right)$.

Proof of the claim in case $2^{\circ}$. If there is no infinite sequence $\left\{x_{i}\right\}_{i} \subset \Xi\left(M_{\varepsilon_{i}}\right) \cap$ $M_{1}\left(\varepsilon_{0}\right)$, then there exists an index $n$ such that for any $i>n, \Xi\left(M_{\varepsilon_{i}}\right) \subset M_{2}(1)$. Again by Courant's Theorem, for each $i>n, M_{\varepsilon_{i}} \backslash \Xi\left(M_{\varepsilon_{i}}\right)$ consists of two connected disjoint open subsets $M_{\varepsilon_{i}}^{+}, M_{\varepsilon_{i}}^{-}$of $M_{\varepsilon_{i}}$, defined as $M_{\varepsilon_{i}}^{+(-)}=\left\{x \in M_{\varepsilon_{i}} ; f_{1}^{1, \varepsilon_{i}}(x)>\right.$ $(<) 0\}$. As a result, $M_{1}\left(\varepsilon_{0}\right) \subset M_{\varepsilon_{i}}^{+}$, or $M_{1}\left(\varepsilon_{0}\right) \subset M_{\varepsilon_{i}}^{-}$, for all $i>n$. The convergence $f_{1}^{1, \varepsilon_{i}} \longrightarrow f_{1}$ in $C^{0}\left(M_{1}\left(\varepsilon_{0}\right)\right)$ implies that $f_{1}$ has to be either nonnegative on $M_{1}\left(\varepsilon_{0}\right)$ or nonpositive. But this cannot happen, because $\Xi\left(M_{1}\right) \subset M_{1}\left(\varepsilon_{0}\right)$ and $f_{1}$ has to change sign on $M_{1}\left(\varepsilon_{0}\right)$.

\section{Conclusions}

Lemma 2.7 "ties" the nodal set of an eigenfunction to dividing curves of an induced contact structure. It would be very interesting to show that dividing curves of an arbitrary contact structure are nodal sets in some suitably adapted metric. 
Problem 4.1. Let $(M, \xi)$ be a contact 3-manifold and $\Sigma$ an embedded convex surface. Can one adapt a metric $g$ to $\xi$ in a tubular neighborhood of $\Sigma$ so that the dividing curves correspond to nodal curves of a $\Delta_{\Sigma}$-eigenfunction on $\left(\Sigma, g_{\Sigma}\right)$ ?

Since isotopic contact structures have isotopic dividing curves, in the formulation of the above problem we can allow the distribution $\xi$ to vary up to isotopy. If the answer to Problem 4.1 is "yes", one could rephrase questions about nodal curves in terms of dividing curves and vice versa. Especially, one could try to address questions of the following type using topological techniques.

Problem 4.2. What kind of nonsingular nodal curves are admissible by a given compact orientable surface $\Sigma$ ? In other words, can we realize a given collection of embedded closed curves $\left\{\Gamma_{i}\right\}_{i}$ on $\Sigma$ as nodal curves (possibly up to isotopy) for some Riemannian metric $g_{\Sigma}$ on $\Sigma$ ?

There are some obvious restrictions on the family $\left\{\Gamma_{i}\right\}_{i}$, e.g. it must divide the surface $\Sigma$. To generate examples of such families, we could adapt the technique from Section 3. It seems possible to start with a generic nodal set embedded into a 2-sphere $S^{2}$ and "implant" a finite number of small handles (as pictured on Figure 1), which collapse to centers of attaching circles, as a parameter $\varepsilon \rightarrow 0$. By similar reasoning as in Section 3.3 we could find a "generic" metric on a surface obtained during this process, with the nodal set isotopic to the initial nodal set on $S^{2}$. One could also start with an arbitrary orientable surface and carry out the implanting procedure. It may also be interesting to relate this to gluing of contact structures along convex surfaces with Legendrian boundary (see 19]).

In the context of Giroux's Theorem 2.4 and the active search for the classification result for tight contact structures, it may be interesting to address the following problem.

Problem 4.3. Find conditions on the metric of a closed surface so that all the nodal curves of $\Delta_{\Sigma^{-}}$-eigenfunctions are homotopically nontrivial.

The hyperbolic case would seem to be of special interest here.

\section{ACKNOWLEDGMENTS}

Advice and comments from M. Dillon, R. Ghrist, J. Landsberg, M. Symington, and A. Swiech were greatly helpful. This paper is part of the author's Ph.D. thesis work under the supervision of R. Ghrist.

\section{REFERENCES}

[1] G. Alessandrini. Nodal lines of eigenfunctions of the fixed membrane problem in general convex domains. Comment. Math. Helv., 69(1):142-154, 1994. MR1259610 (95d:35111)

[2] C. Anné. Perturbation du spectre $X-T U B^{\epsilon} Y$ (conditions de Neumann). In Séminaire de Théorie Spectrale et Géométrie, No. 4, Année 1985-1986, pages 17-23. Univ. Grenoble I, Saint, 1986. MR 1046060

[3] C. Anné and B. Colbois. Opérateur de Hodge-Laplace sur des variétés compactes privées d'un nombre fini de boules. J. Funct. Anal., 115(1):190-211, 1993. MR.1228148(94i:58197)

[4] T. Aubin. Some nonlinear problems in Riemannian geometry. Springer Monographs in Mathematics. Springer-Verlag, Berlin, 1998. MR1636569 (99i:58001)

[5] S. Bando and H. Urakawa. Generic properties of the eigenvalue of the Laplacian for compact Riemannian manifolds. Tôhoku Math. J. (2), 35(2):155-172, 1983. MR0699924 (84h:58146)

[6] S. Y. Cheng. Eigenfunctions and nodal sets. Comment. Math. Helv., 51(1):43-55, 1976. MR0397805 (53:1661) 
[7] S. S. Chern and R. S. Hamilton. On Riemannian metrics adapted to three-dimensional contact manifolds. In Workshop Bonn 1984 (Bonn, 1984), volume 1111 of Lecture Notes in Math., pages 279-308. Springer, Berlin, 1985. MR0797427 (87b:53060)

[8] Y. Eliashberg. Classification of overtwisted contact structures on 3-manifolds. Invent. Math., 98(3):623-637, 1989. MR1022310 (90k:53064)

[9] J. Etnyre. Tight contact structures on lens spaces. Commun. Contemp. Math., 2(4):559-577, 2000. MR.1806947 (2001k:57017)

[10] J. Etnyre and R. Ghrist. Contact topology and hydrodynamics. I. Beltrami fields and the Seifert conjecture. Nonlinearity, 13(2):441-458, 2000. MR1735969 (2001b:76008)

[11] P. Freitas. Closed nodal lines and interior hot spots of the second eigenfunction of the Laplacian on surfaces. Indiana Univ. Math. J., 51(2):305-316, 2002. MR.1909291 (2003d:58045)

[12] D. Gauld. Differential topology, An introduction, volume 72 of Monographs and Textbooks in Pure and Applied Mathematics. Marcel Dekker Inc., New York, 1982. MR0680937 (84k:57013)

[13] R. Ghrist and R. Komendarczyk. Overtwisted energy-minimizing curl eigenfields. preprint, arXiv:math.SG/0411319.

[14] D. Gilbarg and N. Trudinger. Elliptic partial differential equations of second order, volume 224 of Grundlehren der Mathematischen Wissenschaften [Fundamental Principles of Mathematical Sciences]. Springer-Verlag, Berlin, second edition, 1983. MR0737190 (86c:35035)

[15] E. Giroux. Structures de contact sur les variétés fibrées en cercles audessus d'une surface. Comment. Math. Helv., 76(2):218-262, 2001. MR1839346(2002c:53138)

[16] M. Hoffmann-Ostenhof, T. Hoffmann-Ostenhof, and N. Nadirashvili. On the nodal line conjecture. In Advances in differential equations and mathematical physics (Atlanta, GA, 1997), volume 217 of Contemp. Math., pages 33-48. Amer. Math. Soc., Providence, RI, 1998. MR:1605269 (99c:35045)

[17] K. Honda. On the classification of tight contact structures. I. Geom. Topol., 4:309-368 (electronic), 2000. MR.1786111 (2001i:53148)

[18] K. Honda. On the classification of tight contact structures. II. J. Differential Geom., 55(1):83143, 2000. MR1849027 (2002g:53155)

[19] K. Honda, W. Kazez, and G. Matić. Convex decomposition theory. Int. Math. Res. Not., (2):55-88, 2002. MR1874319 (2002k:57064)

[20] R. Komendarczyk. . Ph.D. thesis - in preparation.

[21] J. Martinet. Formes de contact sur les variétés de dimension 3. In Proceedings of Liverpool Singularities Symposium, II (1969/1970), pages 142-163. Lecture Notes in Math., Vol. 209, Springer, Berlin, 1971. MR0350771 (50:3263)

[22] A. Melas. On the nodal line of the second eigenfunction of the Laplacian in $\mathbf{R}^{2}$. J. Differential Geom., 35(1):255-263, 1992. MR 1152231(93g:35100)

[23] L. Payne. Isoperimetric inequalities and their applications. SIAM Rev., 9:453-488, 1967. MR0218975 (36:2058)

[24] J. Rauch and M. Taylor. Potential and scattering theory on wildly perturbed domains. $J$. Funct. Anal., 18:27-59, 1975. MR0377303 (51:13476)

[25] J. Roe. Elliptic operators, topology and asymptotic methods, volume 395 of Pitman Research Notes in Mathematics Series. Longman, Harlow, second edition, 1998. MR1670907 (99m:58182)

[26] S. Rosenberg. The Laplacian on a Riemannian manifold, An introduction to analysis on manifolds, volume 31 of London Mathematical Society Student Texts. Cambridge University Press, Cambridge, 1997. MR1462892 (98k:58206)

[27] J. Takahashi. Collapsing of connected sums and the eigenvalues of the Laplacian. J. Geom. Phys., 40(3-4):201-208, 2002. MR1866987 (2002h:58046)

[28] K. Uhlenbeck. Generic properties of eigenfunctions. Amer. J. Math., 98(4):1059-1078, 1976. MR0464332 (57:4264)

School of Mathematics, Georgia Institute of Technology, Atlanta, Georgia 30332

E-mail address: rako@math.gatech.edu 